\title{
28 Research Square \\ Immune infiltration of MMP14 in Pan-cancer and its prognostic effect on tumor
}

\section{Minde Li}

First Affiliated Hospital of Nanchang University

\section{Shaoyang Li}

First Affiliated Hospital of Nanchang University

\section{Lin Zhou}

First Affiliated Hospital of Nanchang University

\section{Le Yang}

First Affiliated Hospital of Nanchang University

\section{Xiao Wu}

First Affiliated Hospital of Nanchang University

\section{Bin Tang}

First Affiliated Hospital of Nanchang University

\section{Shenhao Xie}

First Affiliated Hospital of Nanchang University

\section{Linchun Fang}

First Affiliated Hospital of Nanchang University

\section{Suyue Zheng}

First Affiliated Hospital of Nanchang University

\section{Tao Hong ( $\sim$ ht2000@vip.sina.com )}

First Affiliated Hospital of Nanchang University https://orcid.org/0000-0002-2693-8991

\section{Research Article}

Keywords: MMP14, pan-cancer analysis, immune infiltration, prognosis, tumor immune

Posted Date: May 25th, 2021

DOl: https://doi.org/10.21203/rs.3.rs-555119/v1

License: (9) This work is licensed under a Creative Commons Attribution 4.0 International License. Read Full License 


\section{Abstract}

Background: Matrix metallopeptidase 14(MMPL4) is a member of the matrix metalloproteinase family, which interacts with tissue metalloproteinase inhibitors (TIMPs), and is involved in normal physiological functions such as cell migration, invasion, metastasis, angiogenesis and proliferation, as well as tumor genesis and progression. However, there has been a lack of relevant reports on the effect of MMP14 on pan-cancer. This study aims to explore the correlation between MMP14 and pan-cancer prognosis, immune infiltration, and the effects of pan-cancer gene mismatch repair (MMR), microsatellite instability (MSI), tumor mutation load (TMB), DNA methylation, and immune checkpoint genes.

Methods: In this study, we used bioinformatics to analyze data from multiple databases, including TCGA, Oncomine and Kaplan-Meier Plotter. We investigated the relationship between the expression of MMP14 in tumors and tumor prognosis, the relationship between MMP14 expression and tumor cell immune infiltration, and the relationship between MMR gene mismatch repair (MMR), microsatellite instability (MSI), tumor mutation load (TMB), DNA methylation, and immune checkpoint genes.

Results: MMP14 expression is highly associated with prognosis of a variety of cancers, tumor immunoinvasion, and has important effects on pan-oncologic mismatch repair (MMR), microsatellite instability (MSI), tumor mutation load (TMB), DNA methylation, and immune checkpoint genes.

Conclusion: MMP14 is highly correlated with tumor prognosis and immunoinvasion, and affects the occurrence and progression of many tumors. All these fully indicate that MMP14 may be a biomarker for the prognosis, diagnosis and treatment of many tumors, and provide a new idea and direction for subsequent tumor immune research and treatment strategies.

\section{Introduction}

Matrix metalloproteinase 14 (MMP14), also called membrane type 1 metalloproteinase (MT1-MMP), is a member of the MT-MMP subfamily.Matrix metalloproteinases are a class of zinc-binding proteinases that contribute to tumor metastasis by degrading the extracellular matrix (ECM) (1).As a member of the first MMPs family to be identified, MMP14 is involved in many biological processes in cells, including proliferation, invasion, vascular production, basement membrane remodeling, etc. $(2,3)$.In general, the vast majority of MMPs are tissue-deficient and have no proenzyme activity, requiring further activation to produce biological activity, but MMP14 is an exception, which does not require additional activation and can be directly present in active form on the cell membrane (4).It has been reported that the increased expression and activity of MMP14 in tumor cells is directly related to the enhanced cell migration ability (5).It has also been reported that as a tumor promoter, MMP14 acts by inhibiting cell adhesion molecules, and tumor necrosis factor-a (TNF-a) is one of its acting factors (6).Studies have shown that MMP14 is involved in the progression of cervical cancer (CC) by promoting angiogenesis, invasion and lymph node metastasis, and it has also been reported that MMP14 overexpression is associated with poor prognosis 
of cervical cancer $(7,8,9)$. However, there is no study on the effect of MMP14 on the immune infiltration and prognosis of pan-tumor cells, which needs to be further improved.

The relationship between tumor and the immune system is complex, and the mechanism of interaction is currently unknown (10). Tumor microenvironment plays an important role in tumor and immune system, in which a large number of cells are composed of immune-infiltrating cells (11).It has long been widely believed that the immune system plays a significant positive role in anti-tumor activities (12), but now a dissenting view has emerged that the immune system can help tumor cells escape predation, and that this effect is attributed to the tumor microenvironment $(13,14,15)$. In recent years, with the deepening of the understanding of immunity, there have been many studies on the value of immunoinfiltrating cells in tumors (16,17).Meanwhile, factors including cytotoxic T-lymphocyte-associated antigen 4 (CTLA4), programmed death-1 (PD-1), and programmed death ligand-1 (PD-L1) have been shown to play an important role in tumor treatment $(18,19)$. However, the proportion of tumors that respond to these immunonode inhibitors is reported to be very small (19). Therefore, it is very important and urgent to strengthen the research on the treatment of immune infiltration microenvironment.

In this study, we used bioinformatics to analyze data from multiple databases, including TCGA, Oncomine, and Kaplan-Meier Plotter.We investigated the relationship between the expression of MMP14 in tumors and tumor prognosis, the relationship between MMP14 expression and tumor cell immune infiltration, and the relationship between MMR gene mismatch repair (MMR), microsatellite instability (MSI), tumor mutation load (TMB), DNA methylation, and immune checkpoint genes.

Results: MMP14 expression is highly associated with prognosis of a variety of cancers, tumor immunoinvasion, and has important effects on pan-oncologic mismatch repair (MMR), microsatellite instability (MSI), tumor mutation load (TMB), DNA methylation, and immune checkpoint genes.

\section{Material And Method}

\section{MMP14 Expression in Human Cancers in ONCOMINE}

In the Oncomine Database (www.oncomine.org), the p-value was set to 0.001 and the fold change was set to 2, then the mRNA expression level of MMP12 in different types of tumors and their adjacent tissues were compared and analyzed.

\section{Prognostic analysis of MMP14 in Kaplan-Meier Plotter database}

Kaplan and Meier Plotter (https://kmplot.com/analysis/) is a can of 21 kinds of cancer, including breast cancer $(n=7830)$, ovarian cancer $(n=2190)$, lung $(n=3452)$ and affect the survival of gastric cancer $(n=$ 1440 ) of visual graphics platform. We obtained clinical information on pan-cancer from the TCGA database $(20,21,22)$.Then Kaplan-Meier used univariate survival analysis to analyze the correlation between overall survival (OS), disease-specific survival (DSS), progression-free interval (PFI), disease-free interval (DFI)(20) and MMP14 expression in patients with pan-cancer. 


\section{Analysis of Immune Infiltration of MMP14 in TIMER Database}

The TIMER (https://cistrome.shinyapps.io/timer/) is a very powerful and practical platform, application which the user can easily to tumor immune research work and the ability to turn the immune and genomics data visualization (23). The data on the platform to date include more than 10,000 samples from 32 cancers of TCGA, with a large number of tumor infiltrating immune cells (24).In this study, we evaluated the relationship between MMP14 expression and the level of immune infiltrate in 32 cancer types ( 6 subsets :B cells, CD4+ T cells, CD8+ T cells, macrophages, neutrophils, and dendritic cells) and tumor purity. The correlation between MMP14 and prognostic immune cell markers was also analyzed to further predict the potential infiltrative immune cell subtypes.Immune genetic marker screening in the R\&D system website (https://www.rndsystems.com/cn/resources/cell-markers/immune-cells),These genetic markers including B cells, CD8 + T cells and dendritic cells, run out of T cells, macrophages, M1 and M2 macrophages, monocytes, macrophages TAMs (tumor associated macrophages and neutrophils, natural killer (NK) cells and follicular helper T cells (Tfh), regulatory cells (Tregs), 1 (Th1) cells, T T auxiliary auxiliary 2 (Th2 cells, T auxiliary 9 (Th9) cells, T helper cells 17 (Th17) and 22 (Th22) T helper cells. The gene expression level was adjusted by log2 TPM.MMP14 is on the $\mathrm{X}$ axis, and the expression scatter plot of the genes of interest on the $Y$ axis can visualize the correlation between MMP14 and various immune gene markers.

\section{Correlation analysis of MMP14 expression with MMR mutation and DNA methylation}

DNA mismatch repair (MMR) can affect tumorigenesis by correcting DNA replication errors and reducing chromosomal rearrangement $(25,26) . \mathrm{MLH} 1, \mathrm{MSH} 2, \mathrm{MSH}$, EpCAM and PMS2 are the five important genes of MMR.We obtained MMR-related data of tumors from the TCGA database, including 5 important genes and MMP14.The expression levels of MMR gene and MMP14 were analyzed by Spearman correlation method.In addition,DNA methylation is an important factor affecting genes.In this study, Spearman correlation method was used to analyze the relationship between the expression levels of DNMT1, DNMT2, DNMT3A and DNMT3B and the expression of MMP14.

\section{Correlation analysis of MMP14 expression with tumor mutation load (TMB) and microsatellite instability (MSI)}

Tumor mutational burden (TMB) is defined as the total amount of DNA mutations produced by Tumor cells $(27,28,29,30)$.In this study, we extracted the somatic cell data (MAF data) from the TCGA database and used the "MAFTools" R package for analysis. Through correlation processing, the total number of exon mutations was obtained, that is, the TMB of the tumor was determined.Microsatellite instability (MSI) is defined as a phenomenon in which nucleotides of repeating DNA fragments are added or lost $(31,32)$.MSI score can be obtained from TCGA database.Then, Spearman analysis was used to analyze the correlation between MMP14 expression and TMB or MSI.

\section{Statistical}


In the analysis of differential expression of MMP14 in tumors and normal tissues, we used the Oncomines database to analyze $P$ values, fold changes, and gene ranks.Meanwhile, in the survival analysis, Kaplan-Meier method was used to analyze the prognosis of patients according to univariate Cox regression analysis and different expression levels of MMP14.Spearman correlation analysis was also used to evaluate the correlation between MMP14 expression and methyltransferase level, MMR gene expression level, and immune checkpoint gene expression. $R>0.20$ was positively correlated, and $P<0.05$ was considered statistically significant.

\section{Results}

\section{Difference analysis of expression of MMP14 in pan-cancer tissues and normal tissues}

In order to analyze the expression level of MMP14 mRNA in normal tissues and tumors, we analyzed relevant data from Oncomine and Timer databases. In the Oncomine database, compared to normal tissue, The results showed that the expression of MMP14 was higher in the Brain and CNS (central nervous system) Cancer, Breast Cancer, Colorectal Carcinoma, Esophageal Cancer, Head and Neck Cancer, Kidney Cancer, Lung Cancer, Melanoma, Ovarian Cancer, Pancreatic Cancer, and Sarcoma (Figure 1a).On the other hand, MMP14 expression was decreased only in the Liver Cancer.

At the same time, we further confirmed the expression of MMP14 in various cancers compared to normal tissues using the TIMER database (Figure 1b). The results showed that the BLCA(Bladder Cancer), BRCA(Breast Cancer), CHOL(Cholangio carcinoma),COAD(Colon adenocarcinoma),ESCA (carcinoma), KIRC (Kidney renal clear cell carcinoma), KIRP (Kidney renal papillary cell carcinoma), LIHC (Liver hepatocellular carcinoma), LUAD ( Lung adenocarcinoma), LUSC (Lung squamous cell carcinoma), READ (Rectum adenocarcinoma), STAD (Stomach adenocarcinoma) and THCA (Thyroid carcinoma) tissues were significantly increased compared to the normal tissues. Tumors with reduced MMP14 expression compared to normal tissue included: HNSC (Head and Neck squamous cell carcinoma), KICH (Kidney Chromophobe), PRAD (Prostate adenocarcinoma), UCEC(Uterine Corpus Endometrial Carcinoma).

\section{Prognostic role of MMP14 in different tumors}

In order to investigate the prognostic effect of MMP14 on different tumors, we analyzed data from different databases. First, the overall survival(OS), disease-special survival(DSS), disease-free survival(DFS), disease-free interval(DFI) of MMP14 were analyzed by univariate Cox proportional risk regression model in the TCGA database.KM(Kaplan-Meier Plotter) survival curves were accordingly plotted for tumors with significant prognosis (Figure 2).

First, we used gene expression profile data to analyze the relationship between MMP14 expression and tumor prognosis in 33 tumors in the TCGA database.Univariate survival analysis resulted in the forest plot of prognosis for 33 tumors, as shown in Figure 2a. KM plot $2 \mathrm{~b}$ was also plotted with a significant correlation with MMP14 expression. Can see MMP14 low expression in the ACC, BLCA, GBM, HNSC, KIRC, LGG, LIHC, LUAD, MESO, OV, PAAD, and overall survival in THCA positively related to prognosis. 
Because of the statistical data of the tumor death, so we use the TCGA database analysis of 33 kinds of tumor disease specific survival (DSS), get the forest figure and significantly associated with relationship of $\mathrm{KM}$, the result is shown in figure 3, from figure 3 we can find out factors other than the tumor death, lower expression of MMP14 positively related to the prognosis of tumor types with ACC, PAAD, UCEC.

Considering the influence of disease factors on survival analysis results, DFS (disease-free survival) : disease-free survival, which is used to evaluate radical surgery, is generally used to indicate the time from treatment to recurrence.We performed DFS analysis on the above data and obtained the forest map and the KM map with significant prognosis, as shown in Figure 4.It can be seen after radical surgery, the lower expression of MMP14 has significant effects on prognosis of tumor have the ACC, BLCA, BRCA, GBM, KRIC, LGG, LIHC, MESO, OV, PAAD, YHCA, UVM.

PFS is an indicator of how well cancer is treated after palliative care.We also analyzed the PFS, the results data (figure 5), can know from figure 5, after palliative therapy, the lower expression of prognosis of MMP14 effects obvious tumor BLCA, BRCA, KICH, KIRC, LGG, LUSC.

\section{Relationship between expression of MMP14 and immune infiltration in diffuse tumors}

We obtained score data of 6 immunoinfiltrating cells in 33 tumors from the TIMER database.Then the expression of MMP14 was correlated with 6 species (B_cells, CD4_tcells, CD8_t cells, macrophages, dendritic Cells, and Neutrophils), and the correlation between the scores of immune cells was analyzed (Supplement $1,2,3,4$ ). We found that the expression of MMP14 was most significantly associated with the infiltration of immune cells in the 3 tumors: BLCA, BRCA, and COAD (Figure 6).

Immune cells and stromal cells in tumor microenvironment is two main cell types, we was analyzed by using the $\mathrm{R}$ package Estimate each tumor samples immune score and score matrix, it is concluded that 33 MMP14 expression in tumor and immune score (Immunescore) relationship, it can be seen in the correlation was statistically significant $(P<0.05)$, the expression of MMP14 and immune score was positively related to: BLCA, BRCA, from cesg, COAD, DLBC, ESCA, GBM, HNSC,KICH,KIRP, LGG, LIHC, LUAD, LUSC, OV,PAAD,PCPG,PRAD,READ,STAD, THCA. At the same time, ACC, SKCM, TGCT and THYM were negatively correlated (Figure 7).In the relationship between the expression of MMP14 of Stromalscore, the expression of MMP14 was positively correlated with the expression of immune matrix under the condition of statistical significance $(P<0.05)$ :BLCA, BRCA, CESC, COAD, DLBC, ESCA, GBM,HNSC, KICH,KIRC, KIRP, LAML, LGG, LIHC, LUAD, LUSC, MESO, OV, PAAD, PCPG, PARD, READ, SARC, STAD TGTC, THCA, THYM, USEC,UCS, UVM. Negative correlation was found in SKCM $(P=0.24)$ (Fig. 8). The three tumors with the most significant correlation between MMP14 expression and immune cell score are Stromalscore: BRCA,CESC, and COAD(Figure 9A); Immunescore: COAD; LGG; PCPG (figure 9 b).In conclusion, it can be seen that the expression of MMP14 is associated with immune infiltration.

\section{The expression of MMP14 is associated with immune checkpoint genes in endemic cancer}


From the above results, it can be seen that the expression of MMP14 is correlated with the level of immune infiltration, so we further studied the relationship between MMP14 and 47 common immune checkpoint genes (Figure 10).The results showed that the expression of MMP14 was associated with 41 immune checkpoint genes in PRAD, 36 in LGG, 33 in THCA and 29 in KICK. We also found that the immune checkpoint gene CD276 was significantly associated with MMP14 expression in 28 of 33 tumors.The expression of MMP14 is associated with immune checkpoint genes in endemic cancer, and these results provide further evidence that MMP14 expression is associated with the level of immune invasion.

\section{The expression of MMP14 is associated with gene mismatch repair (MMR) and DNA methylation levels in endemic cancer}

MMR is an important correction factor for base mismatches, base deletions and insertion errors during DNA replication and recombination, and plays an important role in maintaining gene stability (25).MMR genes play a crucial role in maintaining the stability of MMR genes. Deletions of some important MMR genes will lead to major errors in DNA replication and recombination, leading to the occurrence of tumors (26).So, in order to study the expression of MMP14 on the influence of the cancer process, we choose for the MMR of several important genes and expression level of MMP14 this direction, the influence of the results (figure 11. A), we can see from the picture the expression of MMP14 and MMR genes larger tumors have a KICK, correlation KIRC, LGG, LIHC, LUSC, OV, PAAD, STAD, TGCT, THCA, UCEC, is one of the most relevant $L G G$, UCEC.

A growing number of recent studies have shown that DNA methylation plays a role in the progression of numerous tumors $(27,28)$. Therefore, in this study, we evaluated the relationship between four DNA methyltransferases and MMP14, as shown in Figure 11. It can be seen that the expression of MMP14 is highly correlated with four DNA methyltransferases in many tumors, especially SARC, SKCM, STAD, TGCT, UCEC, BRCA, HNSC, KICH, Kirc, LGG, LIHC, MESO, OV, and PAAD.These data suggest that MMP14 can affect the occurrence and progression of many tumors through DNA mismatch repair and DNA methylation.

\section{Relationship between MMP14 expression and tumor mutation load (TMB) and microsatellite instability (MSI) in endemic cancer}

Recently it has been reported that tumor mutation load (TMB) plays an important role as a biomarker in predicting the response to immune checkpoint inhibitors (ICls) $(29,30,31)$, such as PD-1/PD-

L1.Microsatellite instability (MSI), which also occurs in the vast majority of tumors, may also serve as a novel and important biomarker to predict the effects of immune checkpoint inhibitors $(32,33)$, as well as PD-1.In this study, we investigated the relationship between MMP14 and tumor mutation load (TMB) and microsatellite instability (MSI) in endemic cancer.The analysis results showed that the expression of MMP14 was positively related with TMB: THYM, SKCM, SARC, SARC PAAD, LUAD, LGG.In contrast, tumors in which MMP14 expression was negatively correlated with TMB included BLCA,HNSC,LIHC, and PRAD(Figure 12A).In terms of MSI, MMP14 expression was positively correlated with MSI in the following 
tumors: TGCT,SARC and COAD;The tumors in which MMP14 expression was negatively correlated with MSI included UCEC, PRAD (Figure 12B).

\section{Discussion}

As is known to all, the treatment and prevention of cancer is a very significant research direction at present, and pan-cancer analysis is one of the important means, which can compare the differences between different tumors, and has great significance and practical value for the discovery of new markers and new effective therapeutic targets of cancer.So far, there have been studies on pan-cancer analysis by means of gene mutations, driver genes, gene copy number changes, and tumor purity, etc. These efforts have been proved to be of great significance for the treatment and diagnosis of tumors, providing new ideas and perspectives for the treatment and prevention of tumors $(34,35,36,37,38)$.

Numerous studies have shown that the MMPs family is involved in the occurrence and progression of a large number of tumors, and MMPs have been shown to be closely related to metastasis and invasion (39).MMP14 is one of the first known members of MMPs and has been shown to act as an activator of MMP2 $(40,41)$.Therefore, it can be seen that MMP14 plays an extremely important role in tumors, and strengthening the role of MMP14 in pan-cancer is of great importance and significance for the treatment, prevention and diagnosis of cancer.Previous studies have shown that high expression of MMP14 is associated with poor prognosis of gastric cancer, colorectal cancer and liver cancer $(42,43,44)$.It has also been suggested that MMP14 can be used as a biological indicator of tumor prognosis (45).Currently, there are still limited studies on whether MMP14 is related to tumor prognosis or as a biomarker for biological prognosis. Whether MMP14 can be used as a biomarker for tumor prognosis needs further studies to confirm.

In this study, we first analyzed the MMP14 expression in the generic cancer, we found that compared with normal tissue MMP14 expression differed in 23 kinds of cancer, and high expression of MMP14 and tumor MMR, MSI, DNA methylation is closely related to TMB, these are all plays an important role in the progression, and prognosis of tumor.At the same time, we found that overexpression of MMP14 was associated with poorer outcomes in a variety of cancers (OS, DSS, PFI, and DFI).In addition, the expression of MMP14 is closely related to the immunoosmosis and $\mathrm{ICl}$ level of human pan-cancer, especially in LGG, PRAD, THCA and other tumors. The results of this study suggest that MMP14 plays a critical role in tumor immunity and may serve as an important biomarker.

There have been some reports on the effect of MMP14 on tumors. Ye Jin et al. (46) indicated that high expression of MMP14 in resectable hepatocellular carcinoma predicted poor prognosis. Yichen Dong et al. (47) pointed out that the increased expression of MMP14 is associated with poor prognosis in Chinese gastric cancer patients, and that MMP14 plays an important role in the progression and prognosis of gastric cancer and is a convincing biomarker for predicting prognosis in Chinese gastric cancer patients.Guangfei Cui et al. (48) indicated that MMP14 predicted a poor prognosis in patients with colorectal cancer.MMP14 plays an important role in the progression and prognosis of colorectal cancer 
and may be a useful biomarker for predicting survival after colectomy.At the same time, some studies have shown that MMP14 is involved in normal physiological functions and tumor-related processes such as cell migration, invasion, metastasis, angiogenesis and proliferation of tumors $(49,50)$.MMP14 has been reported to be upregulated in some cancers $(51,52,53)$ and to promote invasion and metastasis of cancer cells $(47,52,54)$.In this study, the prognosis of MMP14 in pan-cancer patients was analyzed, including Overall survival(OS), disease-special survival(DSS), disease-free interval(DFI), and progressionfree interval(PFI).(OS) analysis of MMP14 lower expression in the ACC, BLCA, GBM, HNSC, KIRC, LGG, LIHC, LUAD, MESO, OV, PAAD, THCA positively related to the overall survival prognosis;(DSS) analysis showed that the low expression of MMP14 was positively correlated with the prognosis of ACC,PAAD and UCEC.(DFI) analysis showed that the low expression of MMP14 significantly affected the prognosis of the tumors including ACC,BLCA,BRCA,GBM, KRIC,LGG,LIHC,MESO, OV,PAAD, YHCA,UVM;(PFI) analysis of MMP14 lower expression significantly influence on prognosis of tumor have BLCA, BRCA, KICH, KIRC, LGG, LUSC, one of the highest correlation is ACC, BLCA, BRCA, LGG, PAAD, KIRC. These data indicate that MMP14 is highly correlated with tumor prognosis.MMP14 is a potential biomarker for prognosis of pancarcinomas.However, this study did not explore the mechanism of action of MMP14 in pan-cancer, which is a deficiency of this study.

Tumor immune microenvironment (TME) has recently been widely studied and has become an important aspect of tumor therapy. Numerous studies have confirmed that TME plays an important role in tumor invasion, microangiogenesis, tumor proliferation, and even immune escape $(55,56,57)$.Immune cells are widely present in the matrix of TME cells and play an important role in the effect of TME on tumors. Common and important immune cells include neutrophils, natural killer cells, macrophages, dendritic cells, $B$ cells, and $T$ cells. In TME, various immune factors are regulated by various key factors in the process of playing their roles $(58,59,60)$.Moreover, more and more studies have shown that immune cells play an important role in the occurrence and development of many tumors $(61,62,63)$. However, to date, there have been no studies on the role of MMP14 in TME.The results of this study showed that a variety of tumors were associated with immune cell infiltration, among which three tumors were most significantly related: BLCA,BRCA, and COAD.This may provide a new direction and target for the treatment and diagnosis of BLCA,BRCA and COAD.Immune and matrix scores are often used to evaluate the number of infiltrating immune cells in TME (64).In this study, the expression of MMP14 in BLCA, BRCA, CESC, COAD, DLBC, ESCA, GBM, HNSC,KICH, KirP,LGG, LIHC, Luad, LUSC, OV,PAAD, PCPG, PRAD, READ, STAD and THCA was positively correlated with immune cell score and Stromalscore, and the three tumors with the most significant correlation between the expression of MMP14 and immune cell score were Stromalscore:BRCA, from cesg, COAD. Immunescore: COAD; LGG; PCPG.Meanwhile, in this study, MMP14 was found to co-express with 47 immune checkpoint markers in cancer.MMP14 expression was associated with 36 immune checkpoint genes in LGG, 33 in THCA, and 29 in KICK. We also found that the immune checkpoint gene CD276 was significantly associated with MMP14 expression in 28 of 33 tumors. These evidences suggest that MMP14 can modulate the infiltration of immune cells, perhaps by regulating or recruiting immune cells, and thus further play a role in tumor regulation. These results fully prove that MMP14 plays an important role in tumor immunity. 
Studies have shown that MMR plays an important role in maintaining the stability and integrity of the whole genome in normal cells. It is generally believed that the main components of MMR are MLH, MSN and PMS(65).The deletion of MMR gene will have an important effect on tumor cells, which can significantly increase the mutation frequency of related genes in tumor cells. This potential defective biomarker caused by the deletion of MMP gene is called microsatellite instability (MSI)(66).A large number of studies have confirmed that MMR gene deletion and MSI are considered important and sensitive biomarkers for many tumors, which play an important role in tumor target prediction and treatment $(67,68,69)$.At the same time, a new method of predicting tumor occurrence and progress of the other and DNA methylation and tumor mutation load (TMB), including DNA methylation is regarded as a mechanism of epigenetic change a phenomenon $(27,28)$, and the definition of the TMB is a kind of method, the size of the measurement of cancer cell mutation to predict the prognosis of patients with tumor, especially ICls response is of great significance in the treatment of cancer $(70,71,72)$.In this study, tumors with high correlation between MMP14 expression and five MMR genes were Kick, Kirc, LGG, LiHC, LUSC, OV, PAAD, Stad, TGCT, THCA, and UCEC, among which LGG and UCEC had the highest correlation. The expression of MMP14 in many tumors is highly correlated with four DNA methyltransferases, especially SARC, SKCM, STAD, TGCT, UCEC, BRCA, HNSC, KICH, Kirc, LGG, LIHC, MESO, OV, and PAAD.At the same time, the expression of MMP14 was positively correlated with TMB: Thym,SKCM,SARC,PAAD, LUAD,LGG;In contrast, the tumors in which MMP14 expression was negatively correlated with TMB were BLCA,HNSC,LIHC, and PAD.In terms of MSI, MMP14 expression was positively correlated with MSI in the following tumors: TGCT,SARC and COAD;The tumors in which MMP14 expression was negatively correlated with MSI were: UCEC, PAD.All of these data indicate for the first time that MMP14 plays an extremely important role in tumor genesis and progression, and that MMP14 may be an important biomarker for the diagnosis, treatment and prognosis of multiple tumors.

At present, there have been a few studies on MMP14, but this study is the first one that takes MMP14 as a potential target in widespread cancer. At the same time, the results of this study also show that MMP14 has an important effect on the prognosis and immune infiltration of many tumors, which provides a new direction for tumor research.At present, the exploration of MMP14 inhibitors continues, but no breakthrough has been made (73). There are many reasons for this. Strengthening the study on the mechanism of MMP14 on tumors should be a breakthrough to solve this problem, which is also an important deficiency of this paper.

\section{Conclusion}

In conclusion, this study explored the important role of MMP14 in tumor prognosis, and the expression of MMP14 was highly correlated with tumor immunoinvasion, especially with obvious tumors including BRCA, CESC, COAD, and LGG, PCPG. Meanwhile, we demonstrate that MMP14 can affect the occurrence and progression of many tumors through TMB, MMR, MSI and DNA methylation. All these fully indicate that MMP14 may be a biomarker for the prognosis, diagnosis and treatment of many tumors, and provide a new idea and direction for subsequent tumor immune research and treatment strategies. 


\section{Abbreviations}

ACC Adrenocortical carcinoma; BLCA Bladder Urothelial Carcinoma; BRCA Breast invasive carcinoma; CESC Cervical squamous cell carcinoma and endocervical adenocarcinoma; CHOL Cholangio carcinoma; COAD Colon adenocarcinoma; DLBC Lymphoid Neoplasm Diffuse Large B-cell Lymphoma; ESCA Esophageal carcinoma; GBM Glioblastoma multiforme; HNSC Head and Neck squamous cell carcinoma; KICH Kidney Chromophobe; KIRC Kidney renal clear cell carcinoma; KIRP Kidney renal papillary cell carcinoma; LAML Acute Myeloid Leukemia; LGG Brain Lower Grade Glioma; LIHC Liver hepatocellular carcinoma; LUAD Lung adenocarcinoma; LUSC Lung squamous cell carcinoma; MESO Mesothelioma; OV Ovarian serous cystadenocarcinoma; PAAD Pancreatic adenocarcinoma; PCPG Pheochromocytoma and ; Paraganglioma; PRAD Prostate adenocarcinoma; READ Rectum adenocarcinoma; SARC Sarcoma; SKCM Skin Cutaneous Melanoma; STAD Stomach adenocarcinoma; TGCT Testicular Germ Cell Tumors; THCA Thyroid carcinoma; THYM Thymoma; UCEC Uterine Corpus ; Endometrial Carcinoma; UCS Uterine Carcinosarcoma; UVM Uveal Melanoma.

\section{Declarations}

\section{Acknowledgements}

Not applicable.

\section{Authors' contributions}

Conceived and designed the experiments: $\mathrm{TH}$; performed the analysis: MD Ll; organize and collect data: $\mathrm{L}$ Y, XW, SY L, ZL, SY Z, SH X; wrote the manuscript: MD LI and TH; revised the manuscript: TH and BT; reference collection and data management: LC F, SY Z. All authors read and approved the fnal manuscript

\section{Funding}

This work was supported by the National Natural Science Foundation of China (No. 81460381 and No. 82060246), Key research and invention plan of Jiangxi Science and Technology Department (20192BBG70026), Natural Science Foundation of Jiangxi Province (S2020ZRMSB1789).

\section{Availability of data and materials}

Oncomine (www.oncomine.org); Kaplan and Meier Plotter (https://kmplot.com/analysis/); TIMER (https://cistrome.shinyapps.io/timer/); TCGA (https://www.cancer.gov/aboutnci/organization/ccg/research/structural-genomics/tcga)

\section{Ethics approval and consent to participate}

Not applicable. 
Not applicable.

\section{Competing interests}

The authors declare that they have no competing interests.

\section{References}

1. Min, Li, Chun-Xia, et al. The Effects of miR-195-5p/MMP14 on Proliferation and Invasion of Cervical Carcinoma Cells Through TNF Signaling Pathway Based on Bioinformatics Analysis of Microarray Profiling.[J]. Cellular Physiology \& Biochemistry International Journal of Experimental Cellular Physiology Biochemistry \& Pharmacology, 2018.

2. Pahwa, S., Stawikowski, M.J., Fields, G.B., 2014. Monitoring and inhibiting MT1-MMP during cancer initiation and progression. Cancers (Basel) 6, 416-435.

3. Yadav L, Puri N, Rastogi V, Satpute P, Ahmad R and Kaur G. Matrix metalloproteinases and cancer roles in threat and therapy. Asian Pac J Cancer Prev 2014; 15: 1085-1091.

4. Itoh, Y., Seiki, M., 2006. MT1-MMP: a potent modifier of pericellular microenvironment. J. Cell. Physiol. 206, 1-8.

5. 5. Strongin, A.Y., 2010. Proteolytic and non-proteolytic roles of membrane type-1 matrix metalloproteinase in malignancy. Biochim. Biophys. Acta 1803, 133-141.

6. Lin Y, Wang J, Jin W, Wang L, Li H, Ma L, Li Q, Pang T: NHE1 mediates migration and invasion of HeLa cells via regulating the expression and localization of MT1-MMP. Cell Biochem Funct 2012;30:41-46.

7. Tee YT, Liu YF, Chang JT, Yang SF, Chen SC, Han CP, Wang PH, Liao CL: Single-nucleotide polymorphisms and haplotypes of membrane type 1-matrix metalloproteinase in susceptibility and clinical significance of squamous cell neoplasia of uterine cervix in Taiwan women. Reprod Sci 2012;19:932-938.

8. Liu T, Zhang X, Gao S, Jing F, Yang Y, Du L, Zheng G, Li P, Li C, Wang C: Exosomal long noncoding RNA CRNDE-h as a novel serum-based biomarker for diagnosis and prognosis of colorectal cancer. Oncotarget 2016;7:85551-85563.

9. Wang H, Zhang X, Huang L, Li J, Qu S, Pan F: Matrix metalloproteinase-14 expression and its prognostic value in cervical carcinoma. Cell Biochem Biophys 2014;70:729-734.

10. Bindea G, Mlecnik B, Tosolini M, Kirilovsky A, Waldner M, Obenauf AC, et al. Spatiotemporal dynamics of intratumoral immune cells reveal the immune landscape in human cancer. Immunity. 2013; 39: 782-95.

11. Finn, O.J. (2008). Cancer immunology. N. Engl. J. Med. 358, 2704-2715

12. Bindea G, Mlecnik B, Tosolini M, Kirilovsky A, Waldner M, Obenauf AC, et al. Spatiotemporal dynamics of intratumoral immune cells reveal the immune landscape in human cancer. Immunity. 
(2013) 39:782-95. doi: 10.1016/j.immuni.2013.10.003

13. Gajewski TF, Schreiber H, Fu YX. Innate and adaptive immune cells in the tumor microenvironment. Nat Immunol. (2013) 14:1014-22. doi: 10.1038/ni.2703

14. Topalian SL, Drake CG, Pardoll DM. Immune checkpoint blockade: a common denominator approach to cancer therapy. Cancer Cell. (2015) 27:450-61. doi: 10.1016/j.ccell.2015.03.001

15. Quail DF, Joyce JA. Microenvironmental regulation of tumor progression and metastasis. Nat Med. (2013) 19: 1423-37. doi: 10.1038/nm.3394

16. Lee KH, Kim EY, Yun JS, Park YL, Do SI. The prognostic and predictive value of tumor infiltrating lymphocytes and hematologic parameters in patients with breast cancer. BMC Cancer. 2018; 18: 938.

17. Lee N, Zakka LR, Mihm MC, Jr., Schatton T. Tumour infiltrating lymphocytes in melanoma prognosis and cancer immunotherapy. Pathology. 2016; 48: 177-87.

18. Gordon SR, Maute RL, Dulken BW, Hutter G, George BM, McCracken MN, et al. PD-1 expression by tumour-associated macrophages inhibits phagocytosis and tumour immunity. Nature. 2017; 545: 495-9.

19. Topalian SL, Drake CG, Pardoll DM. Immune checkpoint blockade: a common denominator approach to cancer therapy. Cancer cell. 2015; 27: 450-61

20. Mizuno, H., Kitada, K., Nakai, K. et al. PrognoScan: a new database for meta-analysis of the prognostic value of genes. BMC Med Genomics 2,18 (2009). doi.org/10.1186/1755-8794-2-18

21. Tang Z, Li C, Kang B, Gao G, Li C, Zhang Z. GEPIA: a web server for cancer and normal gene expression profiling and interactive analyses. Nucleic Acids Res. (2017) 45:W98-W102. doi: 10.1093/nar/gkx247

22. Hou, G.X., Liu, P., Yang, J. and Wen, S. (2017) Mining expression and prognosis of topoisomerase isoforms in non-small-cell lung cancer by using Oncomine and Kaplan-Meier plotter. PLoS ONE 12, e0174515, https://doi.org/10.1371/journal.pone.0174515

23. Taiwen Li, Jingyu Fan, Binbin Wang, Nicole Traugh, Qianming Chen, Jun S. Liu, Bo Li, X. Shirley Liu. TIMER: A web server for comprehensive analysis of tumor-infiltrating immune cells. Cancer Research. 2017;77(21):e108-e110. [DOI][PubMed]

24. Bo Li, Eric Severson, Jean-Christophe Pignon, Haoquan Zhao, Taiwen Li, Jesse Novak, Peng Jiang, Hui Shen, Jon C. Aster, Scott Rodig, Sabina Signoretti, Jun S. Liu, X. Shirley Liu. Comprehensive analyses of tumor immunity: implications for cancer immunotherapy. Genome Biology. 2016;17(1):174. [DOI][PubMed]

25. Li, G. M. (2008). Mechanisms and functions of DNA mismatch repair. Cell Res. 18, 85-98. doi: $10.1038 / \mathrm{cr} .2007 .115$

26. Baretti, M., and Le, D. T. (2018). DNA mismatch repair in cancer. Pharmacol. Ther. 189, 45-62. doi: 10.1016/j.pharmthera.2018.04.004

27. Klutstein, M., Nejman, D., Greenfield, R., and Cedar, H. (2016). DNA methylation in cancer and aging. Cancer Res. 76, 3446-3450. doi: 10.1158/0008-5472.Can-15-3278 
28. Koch, A., Joosten, S. C., Feng, Z., de Ruijter, T. C., Draht, M. X., Melotte, V., et al. (2018). Analysis of DNA methylation in cancer: location revisited. Nat. Rev. Clin. Oncol. 15, 459-466. doi: 10.1038/s41571-018-0004-4

29. Yarchoan, M., Hopkins, A., and Jaffee, E. M. (2017). Tumor mutational burden and response rate to PD-1 inhibition. N. Engl. J. Med. 377, 2500-2501. doi: 10.1056/NEJMc1713444

30. Chan, T. A., Yarchoan, M., Jaffee, E., Swanton, C., Quezada, S. A., Stenzinger, A., et al. (2019). Development of tumor mutation burden as an immunotherapy biomarker: utility for the oncology clinic. Ann. Oncol. 30, 44-56. doi: 10.1093/ annonc/mdy495

31. Samstein, R. M., Lee, C. H., Shoushtari, A. N., Hellmann, M. D., Shen, R., Janjigian, Y. Y., et al. (2019). Tumor mutational load predicts survival after immunotherapy across multiple cancer types. Nat. Genet. 51, 202-206. doi:10.1038/s41588-018-0312-8

32. Dudley, J. C., Lin, M. T., Le, D. T., and Eshleman, J. R. (2016). Microsatellite instability as a biomarker for PD-1 blockade. Clin. Cancer Res. 22, 813-820.doi:10.1158/1078-0432.Ccr-15-1678

33. Hause, R. J., Pritchard, C. C., Shendure, J., and Salipante, S. J. (2016). Classification and characterization of microsatellite instability across 18 cancer types. Nat. Med. 22, 1342-1350. doi: $10.1038 / \mathrm{nm} .4191$

34. Zack, T. I., Schumacher, S. E., Carter, S. L., Cherniack, A. D., Saksena, G., Tabak, B., et al. (2013). Pancancer patterns of somatic copy number alteration. Nat. Genet. 45, 1134-1140. doi: $10.1038 / \mathrm{ng} .2760$

35. Aran, D., Sirota, M., and Butte, A. J. (2015). Systematic pan-cancer analysis of tumour purity. Nat. Commun. 6:8971. doi: 10.1038/ncomms9971

36. Ma, X., Liu, Y., Liu, Y., Alexandrov, L. B., Edmonson, M. N., Gawad, C., et al. (2018). Pan-cancer genome and transcriptome analyses of 1,699 paediatric leukaemias and solid tumours. Nature 555, 371376. doi: 10.1038/nature25795

37. Saghafinia, S., Mina, M., Riggi, N., Hanahan, D., and Ciriello, G. (2018). PanCancer landscape of aberrant DNA methylation across human tumors. Cell Rep. 25, 1066-1080.e8. doi:

10.1016/j.celrep.2018.09.082

38. Priestley, P., Baber, J., Lolkema, M. P., Steeghs, N., de Bruijn, E., Shale, C., et al. (2019). Pan-cancer whole-genome analyses of metastatic solid tumours. Nature 575, 210-216. doi: 10.1038/s41586019-1689-y

39. Sato H, Takino T, Okada Y, et al. A matrix metalloproteinase expressed on the surface of invasive tumour cells. Nature 1994;370:61-5.

40. Dong Y, Chen G, Gao M, Tian X. Increased expression of MMP14 correlates with the poor prognosis of Chinese patients with gastric cancer. Gene 2015;563:29-34.

41. Hui P, Xu X, Xu L, Hui G, Wu S, Lan Q. Expression of MMP14 in invasive pituitary adenomas: relationship to invasion and angiogenesis. Int J Clin Exp Pathol 2015;8:3556-7

42. Dong Y, Chen G, Gao M, Tian X. Increased expression of MMP14 correlates with the poor prognosis of Chinese patients with gastric cancer. Gene. 2015 May 25;563(1):29-34. doi: 
10.1016/j.gene.2015.03.003. Epub 2015 Mar 4. PMID: 25748728.

43. Cui G, Cai F, Ding Z, Gao L. MMP14 predicts a poor prognosis in patients with colorectal cancer. Hum Pathol. 2019 Jan;83:36-42. doi: 10.1016/j.humpath.2018.03.030. Epub 2018 Aug 16. PMID: 30120968.

44. Jin Y, Liang ZY, Zhou WX, Zhou L. High MMP14 expression is predictive of poor prognosis in resectable hepatocellular carcinoma. Pathology. 2020 Apr;52(3):359-365. doi:

10.1016/j.pathol.2020.01.436. Epub 2020 Feb 28. PMID: 32122646.

45. Zhang H, Liu M, Sun Y, Lu J. MMP-14 can serve as a prognostic marker in patients with supraglottic cancer. Eur Arch Otorhinolaryngol. 2009 Sep;266(9):1427-34. doi: 10.1007/s00405-009-0943-6. Epub 2009 Mar 13. PMID: 19283401.

46. Jin Y, Liang ZY, Zhou WX, Zhou L. High MMP14 expression is predictive of poor prognosis in resectable hepatocellular carcinoma. Pathology. 2020 Apr;52(3):359-365. doi: 10.1016/j.pathol.2020.01.436. Epub 2020 Feb 28. PMID: 32122646.

47. Dong Y, Chen G, Gao M, Tian X. Increased expression of MMP14 correlates with the poor prognosis of Chinese patients with gastric cancer. Gene. 2015 May 25;563(1):29-34. doi:

10.1016/j.gene.2015.03.003. Epub 2015 Mar 4. PMID: 25748728.

48. Cui G, Cai F, Ding Z, Gao L. MMP14 predicts a poor prognosis in patients with colorectal cancer. Hum Pathol. 2019 Jan;83:36-42. doi: 10.1016/j.humpath.2018.03.030. Epub 2018 Aug 16. PMID: 30120968.

49. Merchant N,Nagaraju GP,Rajitha B,et al.Matrix metalloproteinases:their functional role in lung cancer[J].Carcinogenesis,2017,38(8):766-780

50. Utispan K,Niyomtham N,Yingyongnarongkul BE,et al.Ethanolic Extract of Ocimum sanctum Leaves Reduced Invasion and Matrix Metalloproteinase Activity of Head and Neck Cancer Cell Lines[J].Asian Pac J Cancer Prev,2020,21(2):363-370

51. Hu Y,Wu F,Liu Y,et al.DNMT1 recruited by EZH2-mediated silencing of miR-484 contributes to the malignancy of cervical cancer cells through MMP14 and HNF1A [J].Clin Epigenetics, 2019,11(1):186

52. Kudelski J,Mlynarczyk G,Darewicz B,et al.Dominative role of MMP-14 over MMP-15 in human urinary bladder carcinoma on the basis of its enhanced specific activity[J].Medicine (Baltimore),2020,99(7):e19224

53. Zhang Q,Lou L,Cai X,et al.Clinical significance of AJUBA, YAP1,and MMP14 expression in esophageal squamous cell carcinoma[J].Int J Clin Exp Pathol,2018,11(12):6018-6024

54. Moch H,Cubilla AL,Humphrey PA,et al.The 2016 WHO Classification of Tumours of the Urinary System and Male Genital Organs-Part A:Renal,Penile,and Testicular Tumours [J].Eur Urol,2016,70(1):93-105

55. Altorki NK, Markowitz GJ, Gao D, Port JL, Saxena A, Stiles B, et al. . The Lung Microenvironment: An Important Regulator of Tumour Growth and Metastasis. Nat Rev Cancer (2019) 19:9-31. $10.1038 /$ s41568-018-0081-9 
56. Sautes-Fridman C, Petitprez F, Calderaro J, Fridman WH. Tertiary Lymphoid Structures in the Era of Cancer Immunotherapy. Nat Rev Cancer (2019) 19:307-25. 10.1038/s41568-019-0144-6

57. Fane M, Weeraratna AT. How the Ageing Microenvironment Influences Tumour Progression. Nat Rev Cancer (2020) 20:89-106. 10.1038/s41568-019-0222-9

58. Xu S. J., Hu H. T., Li H. L., Chang S. (2019). The role of miRNAs in immune cell development, immune cell activation, and tumor immunity: with a focus on macrophages and natural killer cells.Cells 8:1140. 10.3390/cells8101140

59. Chen Y, Meng Z, Zhang L, Liu F. CD2 Is a Novel Immune-Related Prognostic Biomarker of Invasive Breast Carcinoma That Modulates the Tumor Microenvironment. Front Immunol. 2021;12:664845. Published 2021 Apr 23. doi:10.3389/fimmu.2021.664845

60. Zhou B, Gao S. Pan-Cancer Analysis of FURIN as a Potential Prognostic and Immunological Biomarker. Front Mol Biosci. 2021;8:648402. Published 2021 Apr 22. doi:10.3389/fmolb.2021.648402

61. Rabinovich G. A., Gabrilovich D., Sotomayor E. M. (2007). Immunosuppressive strategies that are mediated by tumor cells. Annu. Rev. Immunol. 25 267-296.

10.1146/annurev.immunol.25.022106.141609

62. Beatty G. L., Gladney W. L. (2015). Immune escape mechanisms as a guide for cancer immunotherapy. Clin. Cancer Res. 21 687-692. 10.1158/1078-0432.Ccr-14-1860

63. Osipov A., Saung M. T., Zheng L., Murphy A. G. (2019). Small molecule immunomodulation: the tumor microenvironment and overcoming immune escape. J. Immunother. Cancer 7:224. 10.1186/s40425-019-0667-0

64. Galon J., Angell H. K., Bedognetti D., Marincola F. M. (2013). The continuum of cancer immunosurveillance: prognostic, predictive, and mechanistic signatures. Immunity 39 11-26. 10.1016/j.immuni.2013.07.008

65. Fishel R. (2015). Mismatch repair.J. Biol. Chem. 290 26395-26403. 10.1074/jbc.R115.660142

66. Russo M., Crisafulli G., Sogari A., Reilly N. M., Arena S., Lamba S., et al. (2019). Adaptive mutability of colorectal cancers in response to targeted therapies. Science 366 1473-1480. 10.1126/science.aav4474

67. Dudley J. C., Lin M. T., Le D. T., Eshleman J. R. (2016). Microsatellite instability as a biomarker for PD1 blockade. Clin. Cancer Res. 22 813-820. 10.1158/1078-0432.Ccr-15-1678

68. Hause R. J., Pritchard C. C., Shendure J., Salipante S. J. (2016). Classification and characterization of microsatellite instability across 18 cancer types. Nat. Med. 22 1342-1350. 10.1038/nm.4191

69. Baretti M., Le D. T. (2018). DNA mismatch repair in cancer. Pharmacol. Ther. 189 45-62. 10.1016/j.pharmthera.2018.04.004

70. Yarchoan M., Hopkins A., Jaffee E. M. (2017). Tumor mutational burden and response rate to PD-1 inhibition. N. Engl. J. Med. 377 2500-2501. 10.1056/NEJMc1713444 
71. Chan T. A., Yarchoan M., Jaffee E., Swanton C., Quezada S. A., Stenzinger A., et al. (2019). Development of tumor mutation burden as an immunotherapy biomarker: utility for the oncology clinic. Ann. Oncol. 30 44-56. 10.1093/annonc/mdy495

72. Samstein R. M., Lee C. H., Shoushtari A. N., Hellmann M. D., Shen R., Janjigian Y. Y., et al. (2019). Tumor mutational load predicts survival after immunotherapy across multiple cancer types. Nat. Genet. 51 202-206. 10.1038/s41588-018-0312-8

73. Ling, B.; Watt, K.; Banerjee, S.; Newsted, D.; Truesdell, P.; Adams, J.; Sidhu, S.S.; Craig, A.W. A novel immunotherapy targeting MMP-14 limits hypoxia, immune suppression and metastasis in triplenegative breast cancer models. Oncotarget 2017, 8, 58372-58385.

\section{Figures}

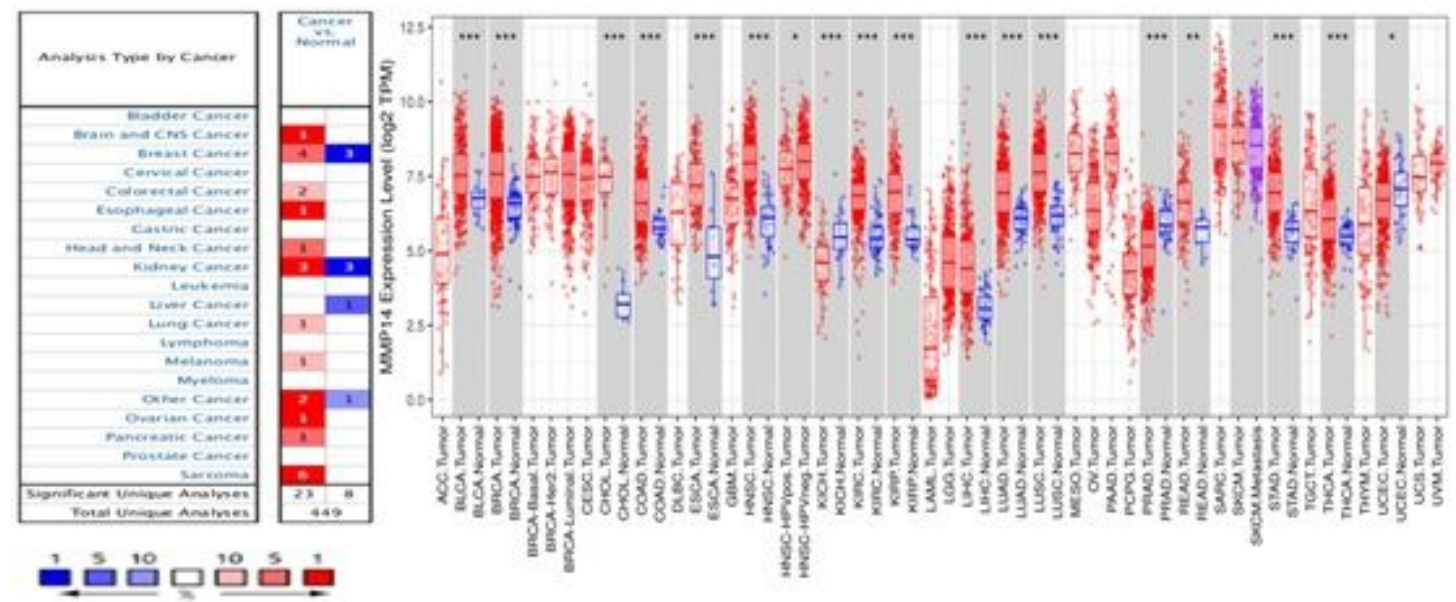

Figure 1

Difference of expression of MMP14 in tumor tissues and normal tissues in pan-cancer. (a) Oncomine database was used to analyze the expression of MMP14 in tumor tissues and normal tissues. The number in each cell is the total amount of the dataset;(b)Analysis and comparison of MMP14 expression in different tumor tissues and normal tissues in TCGA database* $P \otimes 0.05, * * P \otimes 0.01 * * * P \otimes 0.001 \square$ 

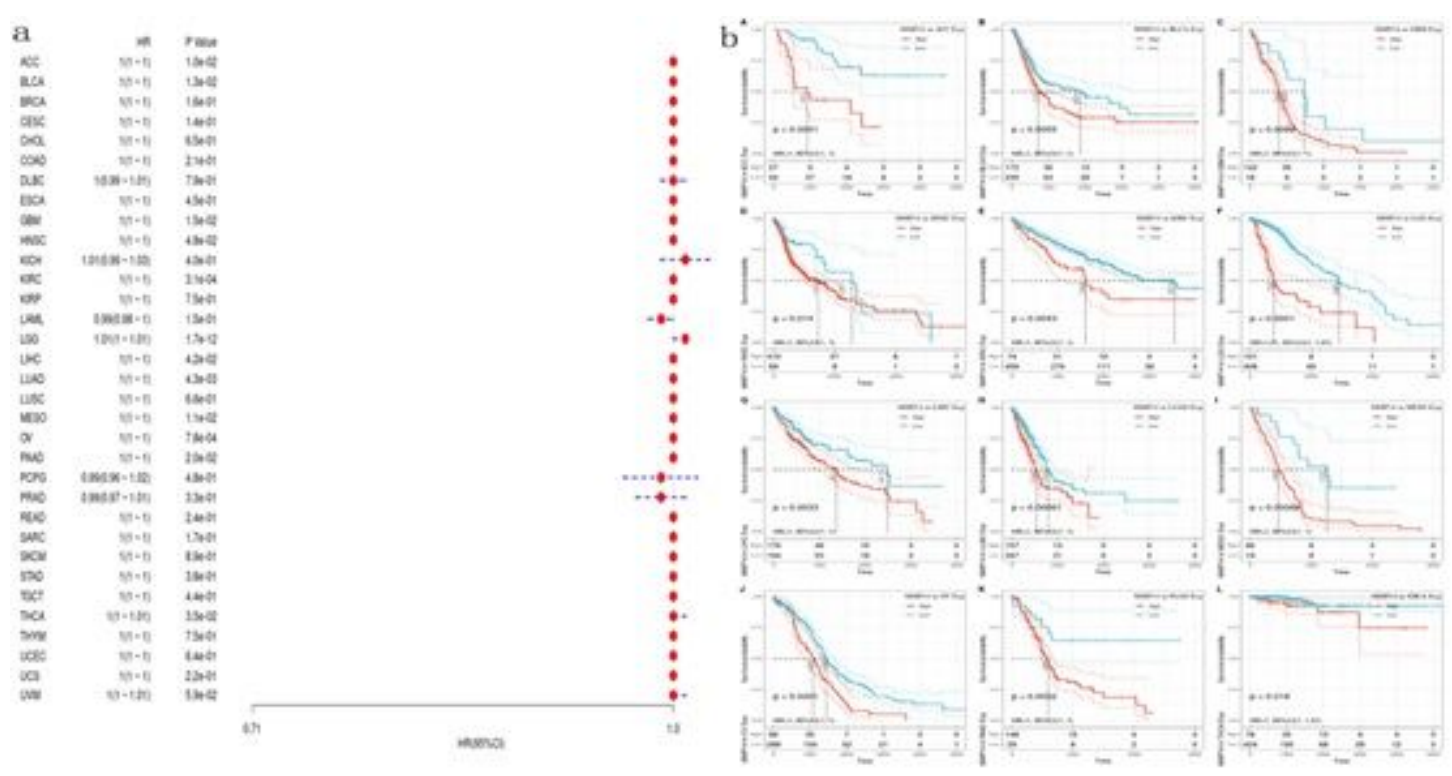

\section{Figure 2}

The relationship between the expression of MMP14 and the Overall Survival (OS) and prognosis of Pan cancer was analyzed. (a) The correlation between MMP14 expression and OS in different cancer types of TCGA was analyzed. The red part represents the risk ratio. Due to the limited sample size, the parameters and hazard ratio could not be calculated with short bars, and the red font indicated $P<0.05$. (b) Kaplan Meier analysis was used to make survival curve for the prognostic effect of MMP14 expression on Pan cancer. OS: overall survival.
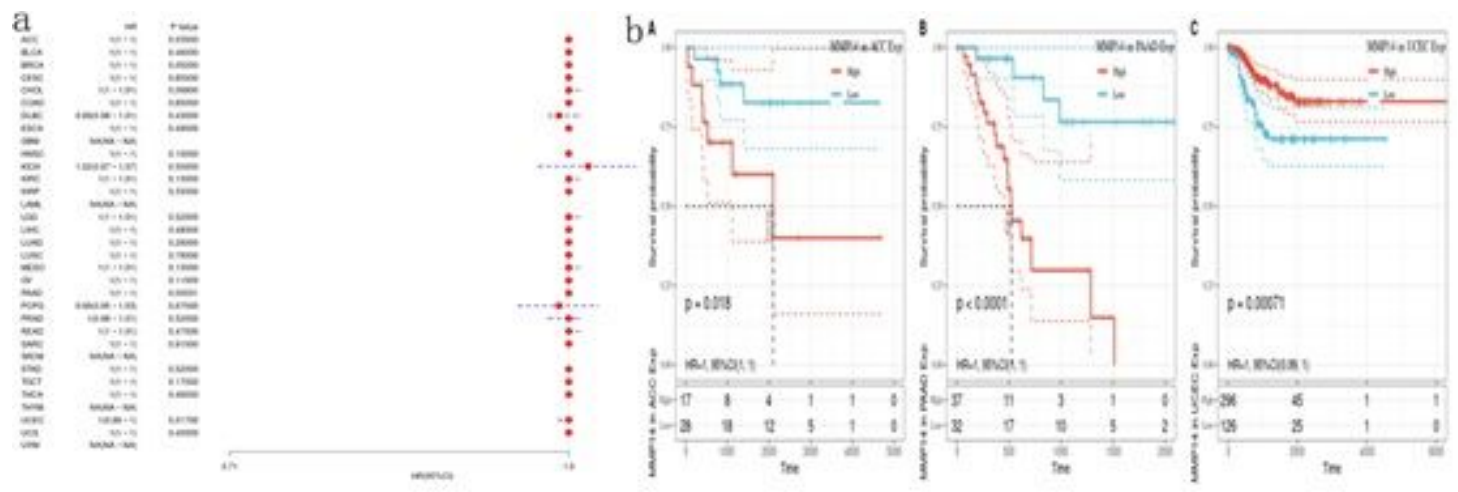

Figure 3

The expression of MMP14 was correlated with the disease-free survival (DFS) and the prognosis was analyzed by DFI. The expression of MMP14 and the overall survival rate of Pan cancer were analyzed by DFI $\mathrm{a}$ ) The correlation analysis of MMP14 expression and DFI in different cancer types of TCGA, the red part represents the risk ratio. Due to the limited sample size, the parameters and hazard ratio could not be calculated with short bars, and the red font indicated $P<0.05$. (b) Kaplan Meier analysis was used to make survival curve for the prognostic effect of MMP14 expression on Pan -cancer. DFI: disease free interval survival. 

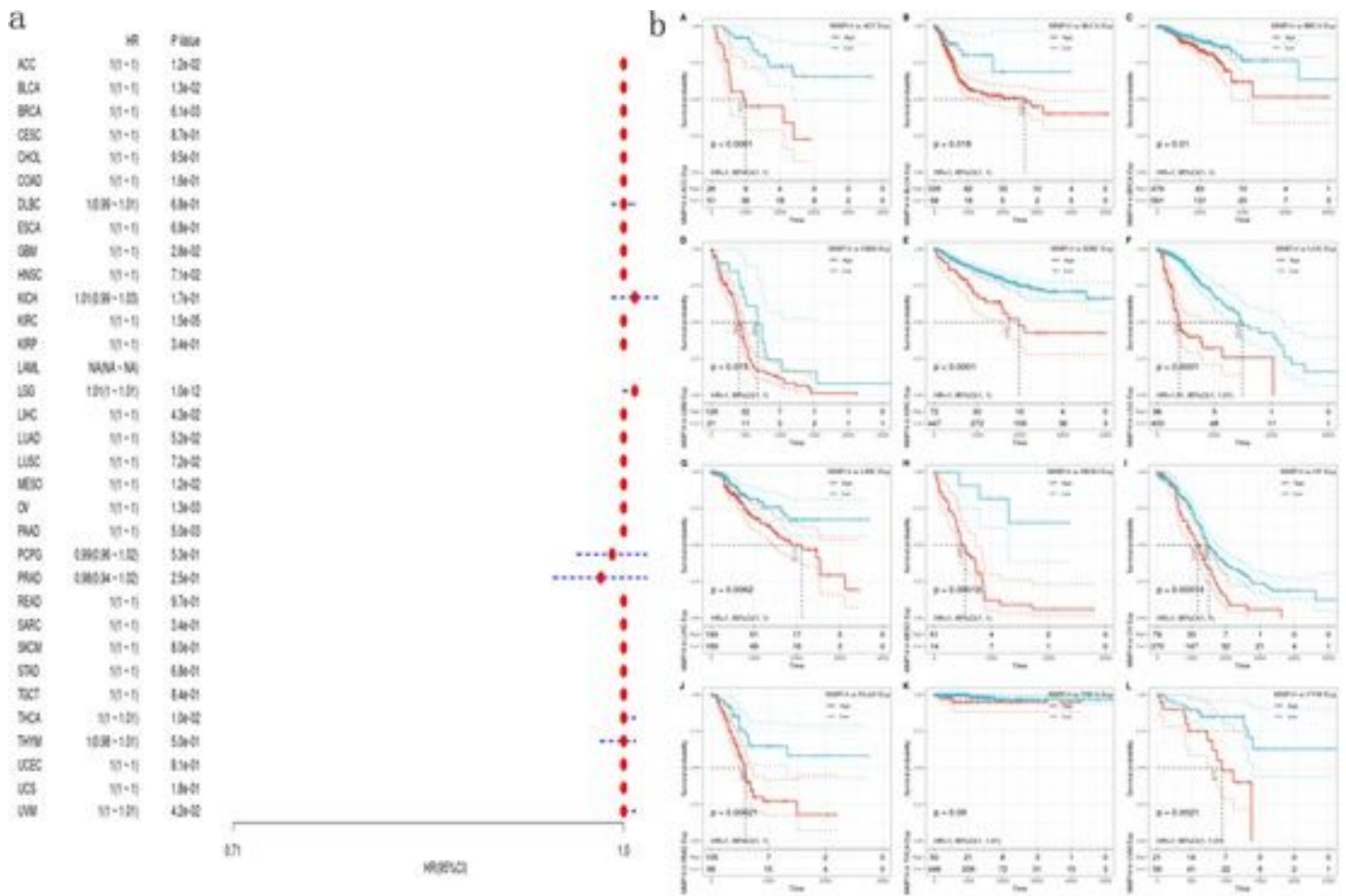

\section{Figure 4}

The expression of MMP14 and the prognosis of DSS were analyzed. The relationship between MMP14 expression and disease-free survival was analyzed by DSS. The expression of MMP14 and the overall

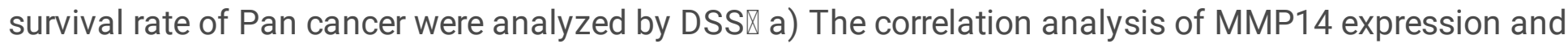
DSS in different cancer types of TCGA, the red part represents the risk ratio. Due to the limited sample size, the parameters and hazard ratio could not be calculated with short bars, and the red font indicated $\mathrm{P}$ $<0.05$. (b) Kaplan Meier analysis was used to make survival curve for the prognostic effect of MMP14 expression on Pan cancer. DSS: disease specific survival.

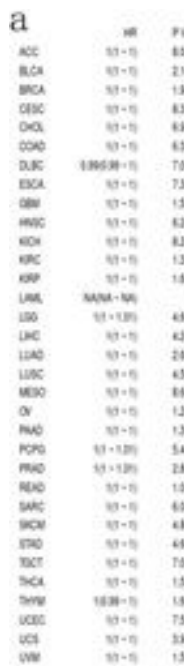
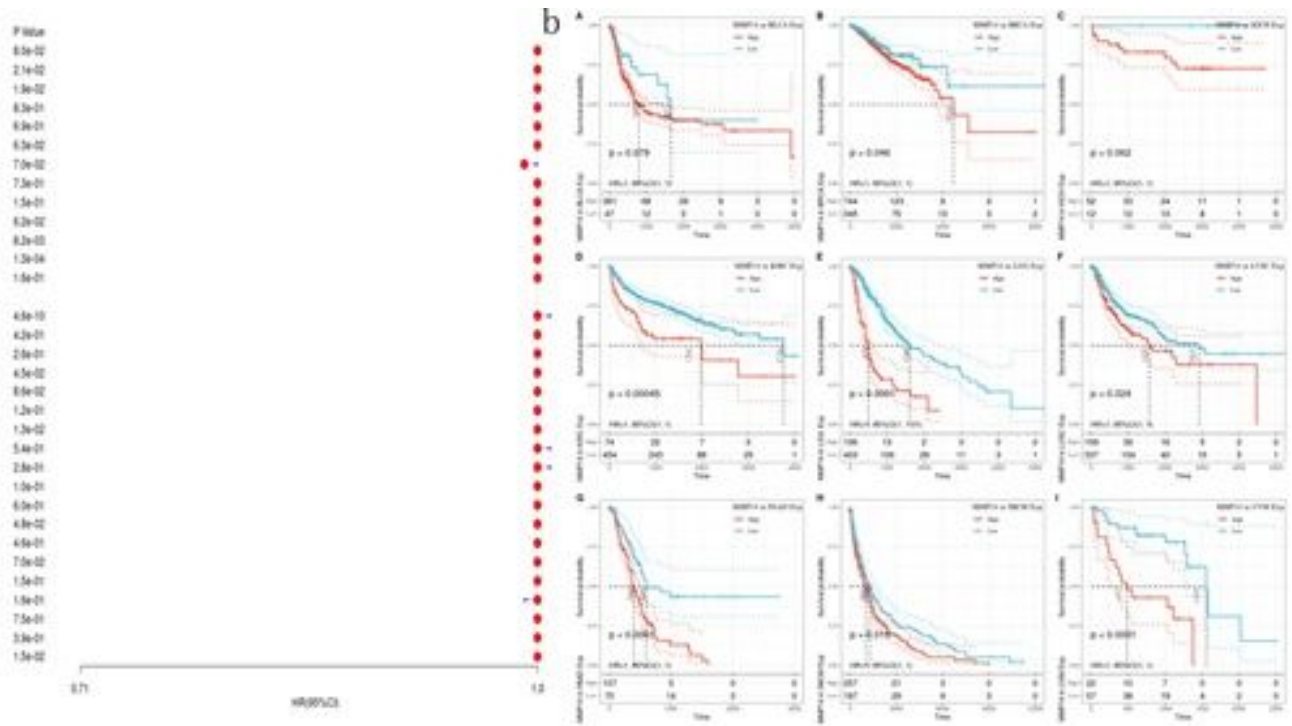

Figure 5 
The relationship between MMP14 expression and PFI prognosis was analyzed. The expression of MMP14 and prognosis of Pan -cancer disease-specific survival were analyzed by PFI. The relationship between MMP14 expression and disease-free survival was analyzed by DSS. The expression of MMP14 and the overall survival rate of Pan -cancer were analyzed by PFI. (a) The correlation analysis of MMP14 expression and PFI in different cancer types of TCGA, the red part represents the risk ratio. Due to the limited sample size, the parameters and hazard ratio could not be calculated with short bars, and the red font indicated $P<0.05$. (b) Kaplan Meier analysis was used to make survival curve for the prognostic effect of MMP14 expression on Pan- cancer. PFI: progression free interval survival.

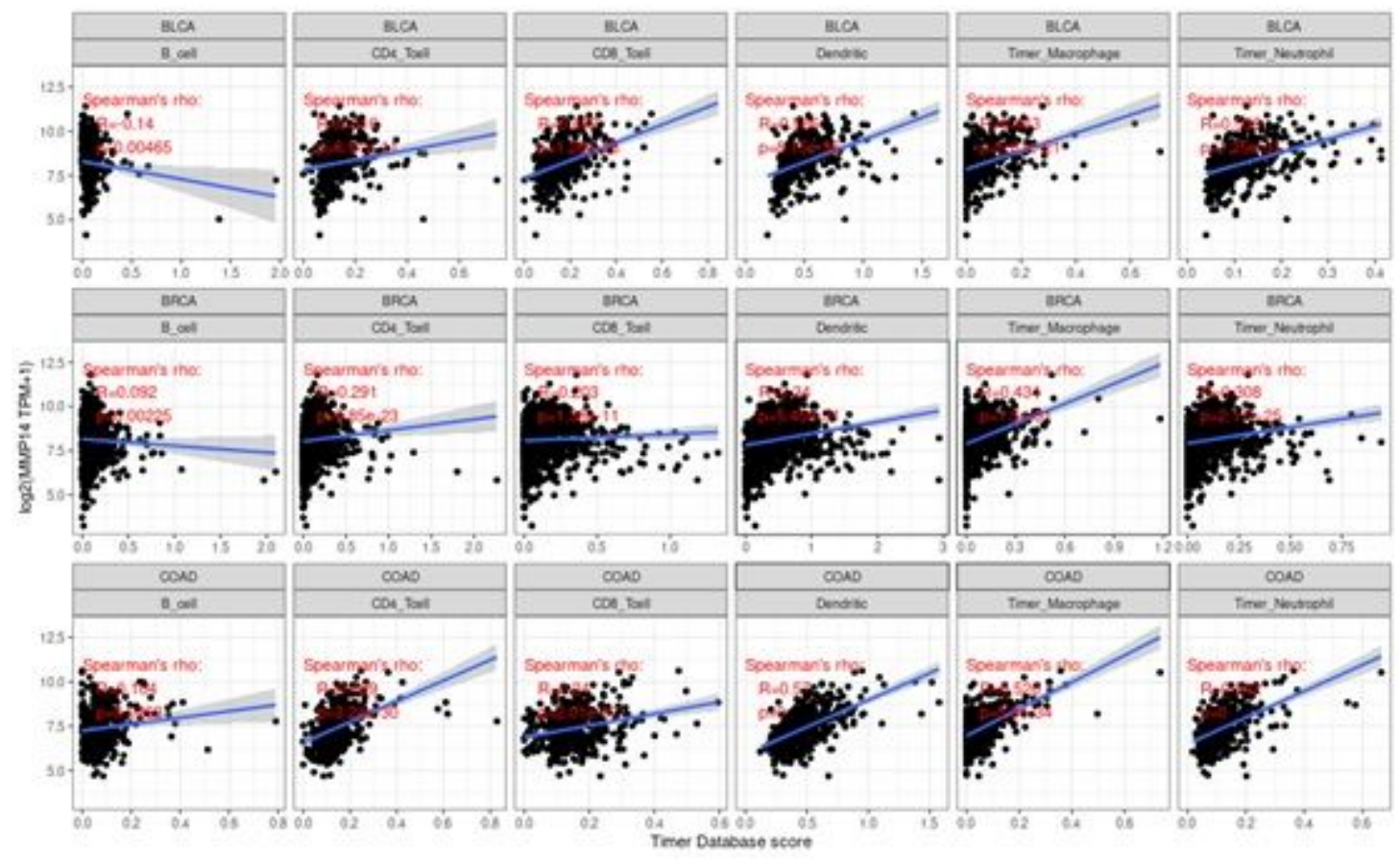

Figure 6

The expression of MMP14 in BLCA, BRCA and COAD was correlated with the level of immune infiltration (B_cells, CD4_tcells, CD8_t cells, macrophages, dendritic Cells, and Neutrophils). Only in BLCA, the expression of MMP14 was negatively correlated with B_cells infiltration, and the others were positively correlated with the 6 kinds of immune cell infiltration. 


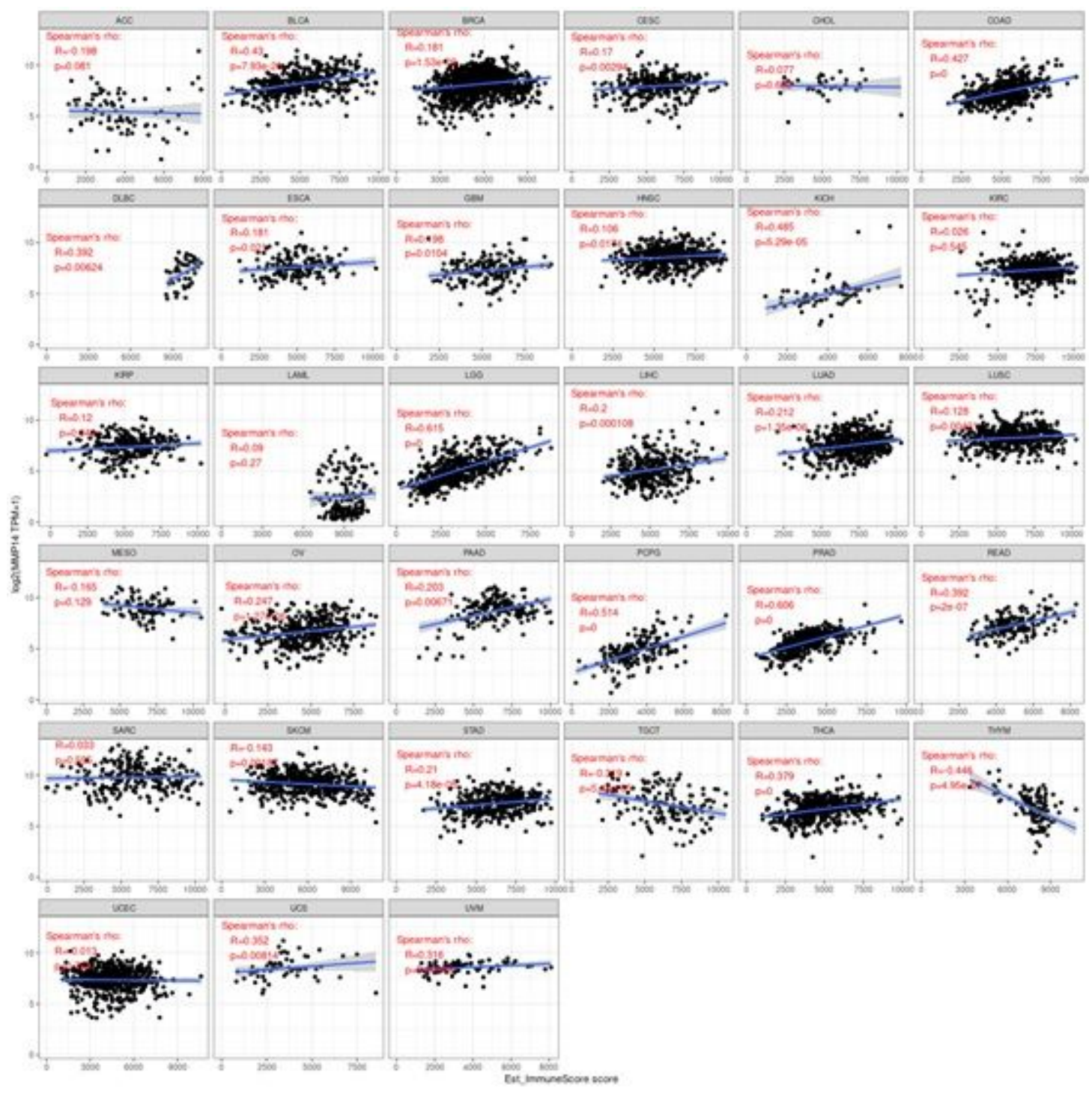

Figure 7

Relationship between the expression of MMP14 and Immune score in Pan-cancer. Only in ACC, SKCM, TGCT and THYM, the expression of MMP14 was negatively correlated with immune cell infiltration, and the others were positively correlated with the immune cell infiltration (In the case of $\mathrm{P}<0.05$ ). 


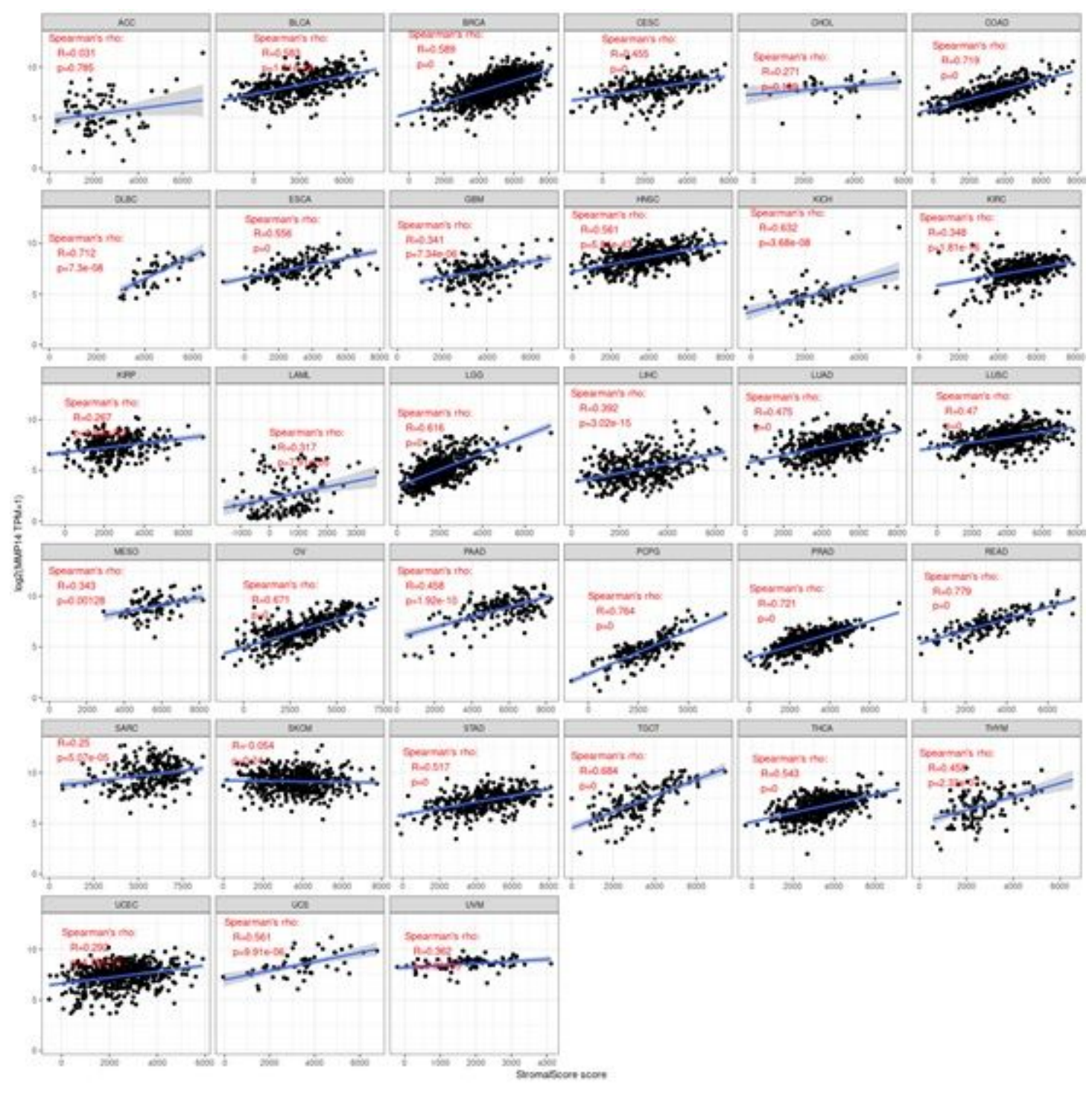

Figure 8

Relationship between the expression of MMP14 and Stroma score in Pan-cancer. Only in SKCM, the expression of MMP14 was negatively correlated with immune cell infiltration, and the others were positively correlated with the immune cell infiltration(In the case of $\mathrm{P}<0.05$ ). 



Figure 9

The 3 Tumors with the highest correlation between MMP14 expression and immune score and stromal score in the pan-cancer analysis. The expression of MMP14 is positively correlated with the infiltration level of these immune cells. 


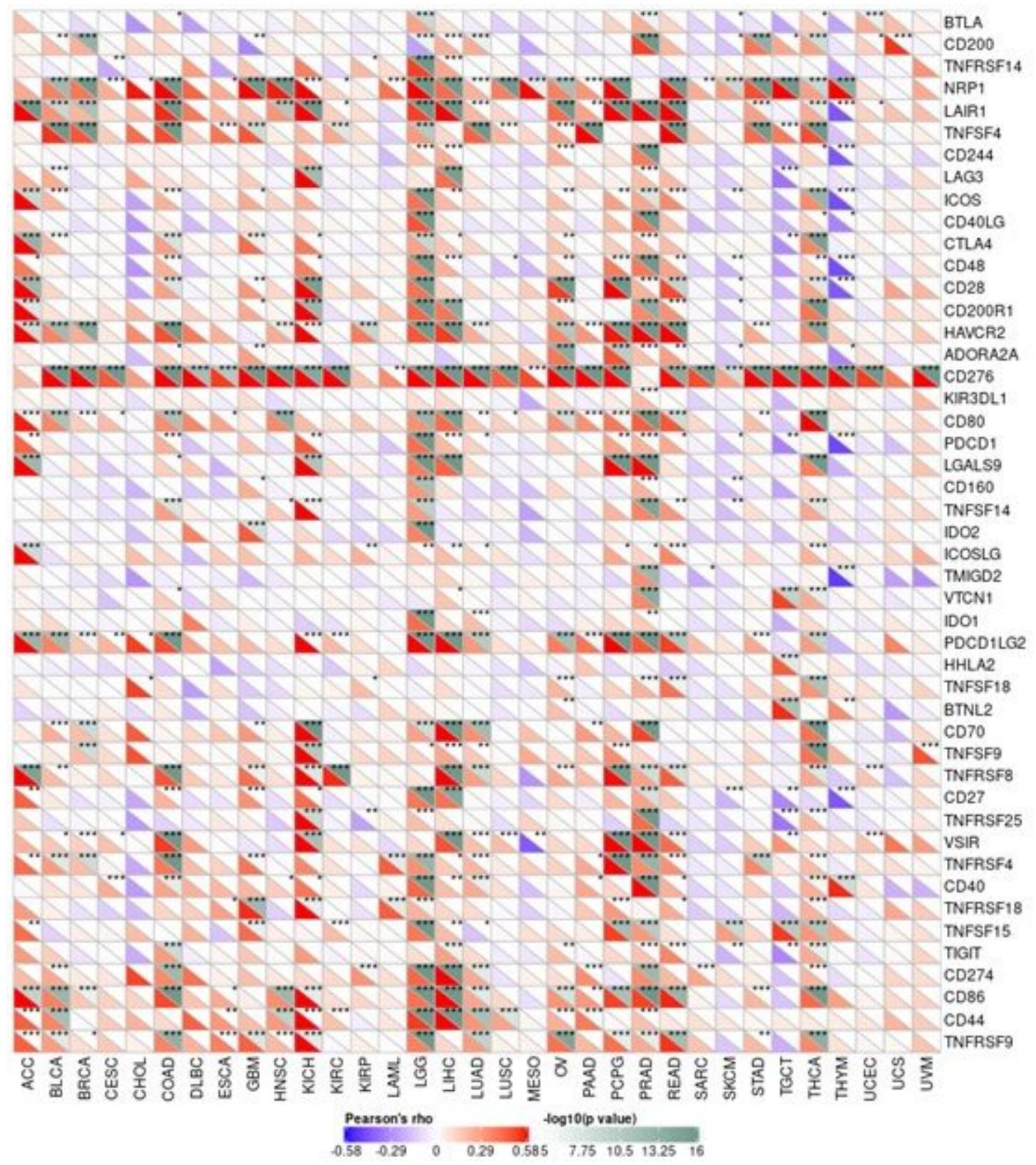

Figure 10

Correlation analysis of MMP14 expression and 47 immune checkpoint genes in endemic cancer. MMP14 was associated with 41 immune checkpoint genes in PRAD, 36 in LGG, 33 in THCA and 29 in KICK. We also found that the immune checkpoint gene CD276 was significantly associated with MMP14 expression in 28 of 33 tumors ( $P<0.05, * * P<0.01 * * P<0.001)$. 


\section{a}

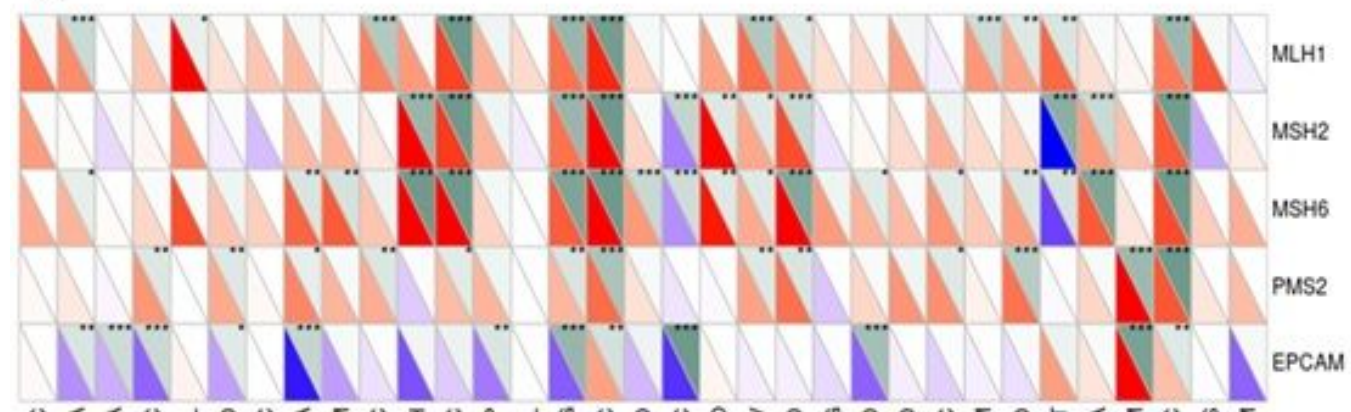

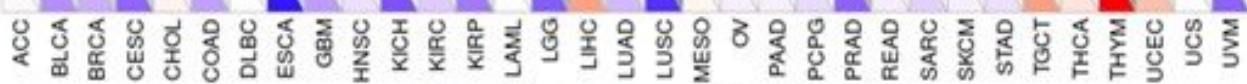



b

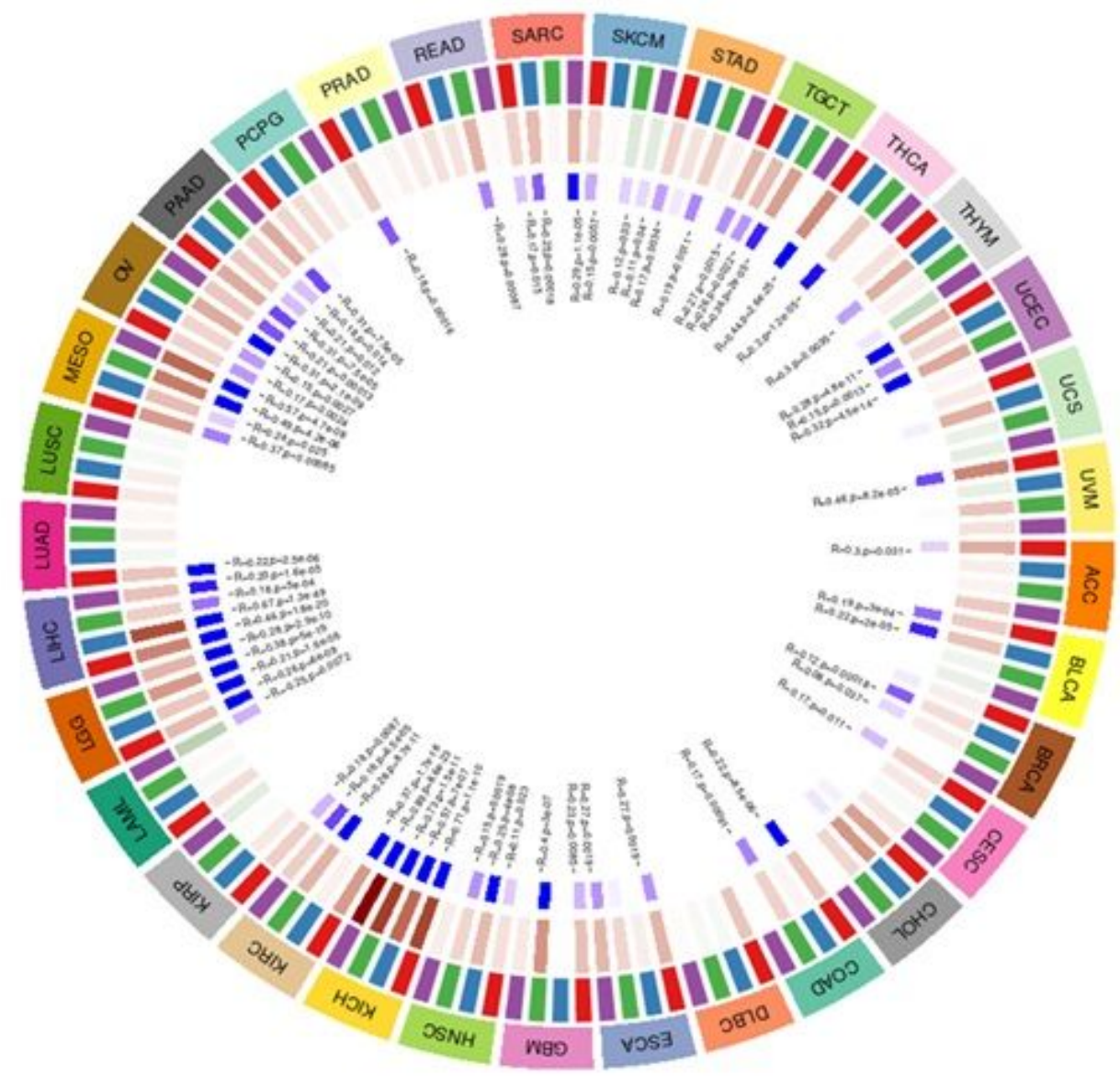

\section{Figure 11}

The expression of MMP14 was correlated with the expression of 5 MMR genes and 4 DNA methyltransferases. (a) Spearman correlation analysis was used to analyze the correlation between MMR gene expression and MMP14 expression in tumors (* $P<0.05$, * $P<0.01 * * P<0.001$ ). (b) Spearman correlation analysis was used to analyze the correlation between four DNA methyltransferases and MMP14 expression 


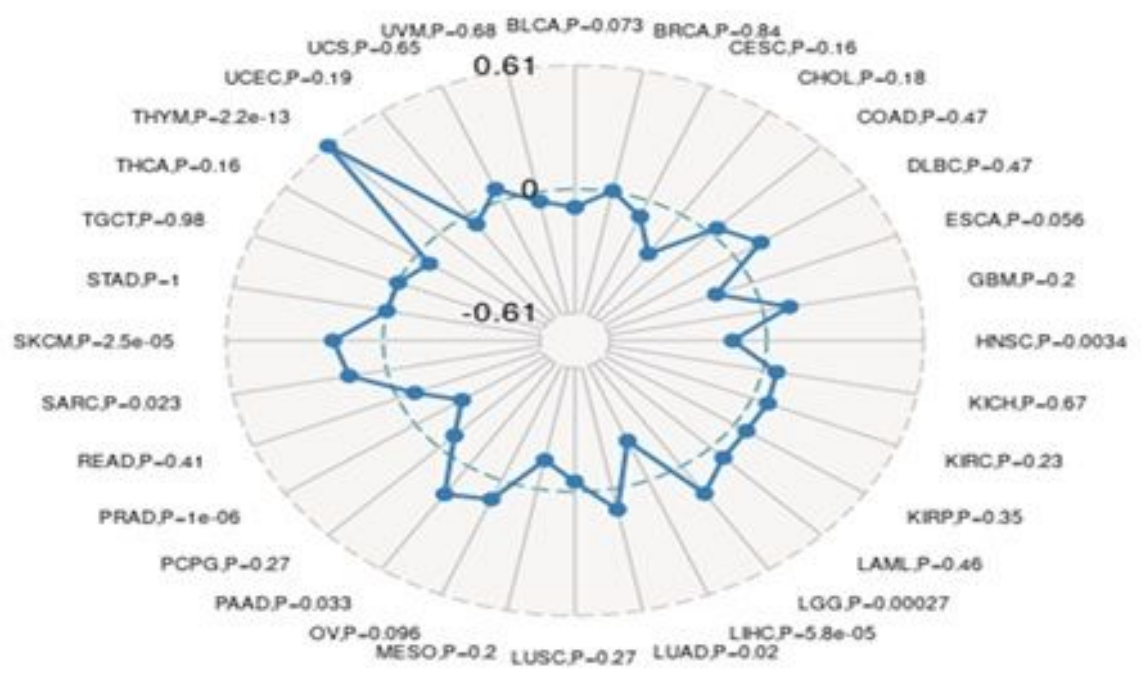

b

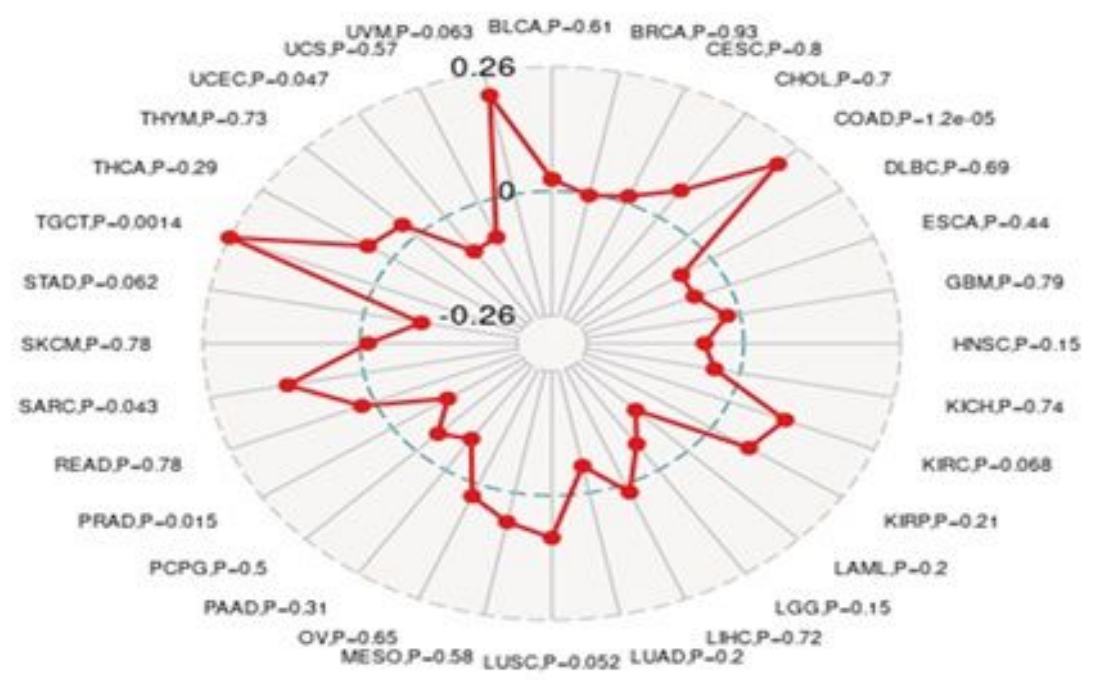

Figure 12

The expression of MMP14 was correlated with TMB and MSIX a) Radar images were used to show the correlation between MMP14 expression and TMB. The black value represents the range and the blue curve represents the correlation coefficient $\mathbb{b}$ b) Radar images were used to show the correlation between MMP14 expression and MSI. The black value represents the range and the red curve represents the correlation coefficient. 